\title{
Oral surgery trends in the last decade
}

\author{
Trends in oral surgery in England and Wales 1991-2000 \\ D. K. Dhariwal, R. Goodey, and J. P. Shepherd Br Dent J 2002; 192: 639-645
}

\section{Objective}

To investigate trends in oral surgery in England and Wales 1991-2000.

\section{Methods}

Oral surgery procedure data were derived from Dental Practice Board and Department of Health Hospital Episode Statistics.

\section{Results}

There was a 6\% increase in minor oral surgery (MOS) procedures, including ordinary extractions, extractions of special difficulty, apicectomies and third molar removals, carried out in the General Dental Services (GDS) but the number of third molars removed fell by 32\% after 1997. General anaesthetics (GA) administered in the GDS fell by 77\% and the number of sedations rose 54\% after 1998. There was concentration of minor oral surgery in practices: in the year 2000, $88 \%$ of practitioners carried out less than five third molar removals. In the Hospital Dental Service (HDS) there was a 98\% increase in day surgery, and a 53\% decrease in ordinary admissions for minor oral surgery. HDS waiting times remained constant over the ten year period.

\section{Conclusions}

The principal trends were substantial decreases in apicectomies, third molar removals after 1997 and GAs after 1998; increases in extractions of special difficulty and concentration of MOS in the GDS. Numbers of ordinary extractions did not change. In the HDS there was a large shift from inpatient to daycase provision which has facilitated expansion of maxillofacial surgery. This is an important example of NHS reconfiguration. Perhaps the most important implication of these changes concerns the place of MOS in vocational training.

\section{IN BRIEF}

- The decade to 2000 was characterised by major changes in NHS oral surgery provision; including a switch from inpatient to day case minor surgery.

- The publication of research and guidelines was followed by reductions in third molar removals in the GDS.

- Numbers of ordinary extractions and hospital waiting times did not change.

- There was a sevenfold increase in GDS claims for extractions of special difficulty in the period $1980-2000$.

- After 1998 there was a major decline in the use of GAs in the GDS but no indications of a similar trend for minor surgery in the HDS.

\section{COMMENT}

This is a carefully conducted and presented study of trends in oral surgery in England and Wales during 1991-2000, which was a period of rapid change. Useful quantitative data are included which provides evidence to support clinicians' perceptions on the changing pattern of oral surgery provision. The effect of research publications and the guidelines from The Faculty of Dental Surgery of The Royal College of Surgeons of England in 1997 is reflected in the decrease in number of third molars removed in the GDS after that time. The change from in-patient to day case general anaesthesia for oral surgery in hospital is quantified, and impacts on the reconfiguration in NHS services. Also seen is the effect of the General Dental Council's guidance to dentists on general anaesthesia in 1998, following which there has been a marked reduction in general anaesthetics in the GDS, with an increase in sedation administration.

The authors highlight several other interesting trends. The marked decrease in apicectomies in the GDS recently may also be due to the increasing success in the re-treatment of root filled teeth by endodontic techniques. Many more 'extractions of special difficulty' are now performed in the GDS, and this trend is likely to continue as the adult population retains their natural dentition into later life. Although the authors could not identify 'specialist surgical dentistry practices', they showed that the provision of minor oral surgery measured in terms of wisdom tooth removal has become concentrated in a smaller number of practices, performed by a small proportion of practitioners. This might indicate the move towards the provision of more surgical dentistry services in the 'high street', as advocated in the Chief Dental Officer's Report on UK Specialist Dental Training in 1995.

These changes also have implications for postgraduate education. The concentration of surgical dentistry in a small number of locations, with $88 \%$ of practitioners carrying out little surgery may mean that a vocational dental practitioner is unable to build on their undergraduate surgical experience. This adds further support to the concept of general professional training, where following VT, a second year as a Senior House Officer in hospital provides a wider training in surgery and other specialty areas. The marked increase in sedation provided by general dental practitioners requires adequate support from appropriate training and updating courses.

The authors acknowledge the limitations of their study, particularly concerning private treatment in primary care where little information is available. They also raise the interesting question of the long term outcome of the current recommendation to leave pathology-free third molars in place. Will there be an epidemic of wisdom tooth problems in elderly patients in the future?

Professor John W Frame

The University of Birmingham 